\title{
Decreased levels of circulating sex hormones as a biomarker of lung cancer in male patients with solitary pulmonary nodules
}

\author{
Tao $\mathrm{Gu}^{1 *}$, Zongmei Wen²*, Shufeng $\mathrm{Xu}^{1}$, Haixia Hua ${ }^{1}$, Zhi Zhang ${ }^{3}$, Tao $\mathrm{Wen}^{4}$, Zhanzhao $\mathrm{Fu}^{1 \#}$, Xin $\mathrm{Lv}^{2}$
}

1. Department of Oncology, First Hospital of Qinhuangdao, Qinhuangdao 066000, Hebei Province, P.R. China

2. Department of Anesthesiology, Shanghai Pulmonary Hospital, Tongji University School of Medicine, Shanghai 200433, P.R. China

3. Department of Oncology, Tangshan Worker's Hospital, Tangshan 063000, Hebei Province, P.R. China

4. Beijing Youan Hospital affiliated with Capital Medical University, Beijing 100069, P.R. China

*Tao Gu and Zongmei Wen contributed equally to this work.

\begin{abstract}
Background: An early differentiation of malignant from benign solitary pulmonary nodules (SPNs) is essential for management and prognosis of lung cancer.

Objectives: Here we investigated whether measurement of circulating sex hormones could be useful for an early detection of malignancy among patients with SPNs.

Methods: We recruited 47 patients with malignant SPNs, 45 patients with benign SPNs, and 32 healthy persons. Testosterone, estradiol, and progesterone were measured. Carcinoembryonic antigen (CEA) as well as TNF- $\boldsymbol{\alpha}$, IL-1 and IL-6 were also measured.

Results: We found that sex hormones were decreased significantly in patients with malignant SPNs, as compared to patients with benign SPNs and healthy controls $(\mathrm{P}<0.05)$. Sex hormones levels showed a trend to decline in patients with benign SPNs as compared to normal controls, but the difference was not statistically significant $(\mathrm{P}>0.05)$. CEA levels were only abnormally elevated in eight patients with lung adenocarcinoma. The inflammatory cytokines were remarkably higher in both patients than in normal controls. However, there was no statistical difference in these cytokines among patients.

Conclusions: The reduced sex hormones levels seemed to be uniquely associated with lung cancer. Therefore, measurement of sex hormones may have clinical potential in the diagnosis of malignancy in patients with SPNs.
\end{abstract}

Keywords: solitary pulmonary nodules (SPNs), sex hormones, lung cancer, biomarkers

African Health Sciences 2014; 14(2):356-363

DOI: http://dx.doi.org/10.4314/ahs.v14i2.10

\section{Introduction}

Solitary pulmonary nodules (SPNs), defined as spherical lesions that measure up to $3 \mathrm{~cm}$ in diameter and that are completely surrounded by pulmonary parenchyma $(1,2)$, are quite commonly seen in clinical practice. Generally, SPNs are discovered incidentally through chest X-rays or computerized tomography (CT) scans that are performed for unrelated purposes(3). It is projected

\section{Corresponding author:}

Xin Lv,

Department of Anesthesiology, Shanghai Pulmonary Hospital, Tongji University School of Medicine, Shanghai 200433, P.R. China

Email:(xinlvg@126.com); or

Zhanzhao Fu, Department of Oncology, First

Hospital of Qinhuangdao, Qinhuangdao 066000, Hebei Province, P.R. China

Email: springmaze@gmail.com that around half of heavy smokers over the age of 50 may have SPNs. The incidence of cancer in patients with SPNs ranges from 10 to 70 percent(4). The early differentiation of malignant from benign SPNs is very critical because it represents a totally different way of management and prognosis(1,3). Benign SPNs are most commonly caused by chronic smoking, infections, intrapulmonary lymph node, and vascular abnormalities(5). In most cases, benign SPNs require no treatment. Malignant SPNs, however, generally represent an early stage of lung cancer or metastatic cancers that have spread to the lung from other regions of the body(6). It is known that lung cancer is the most common cause of cancer-related deaths around the world, accounting for more deaths annually than liver, colon, breast, and prostate cancers(7). The survival rates of lung cancer remain frustratingly low, with a $15 \%$ of 5 -year survival rate, whereas the early diagnosis and proper management of lung cancer may lead to an increase in 5-year survival rates up to $70-80 \%(7,8)$. Therefore, it is critical to improve the early differentiation of malignant from benign SPNs 
in patients. Nevertheless, the asymptomatic nature of SPNs makes for a significant clinical challenge in diagnosing and differentiating between malignant and benign $\operatorname{SPNs}(9,10)$.

For accurate diagnosis of SPNs, it usually includes observation with serial chest X-rays, CT scan, transthoracic needle biopsy, and surgical resection(1). So far, chest X-ray is conducted to identify SPNs as a routine screening test, which avoids unnecessary surgery in case of benign disease but delays appropriate diagnosis and management, when malignancy really exists. Furthermore, serial chest X-rays may lead to unnecessary radiation exposure, anxiety, cost, and low accuracy. CT or positron emission tomography (PET) scan is more sensitive and more specific than chest X-ray, and is used to do further imaging when some SPNs are seen on chest X-ray $(1,11)$. However, the widespread use and improved sensitivity of CT or PET dramatically increase the number of SPNs seen in asymptomatic individuals. Only a small fraction of SPNs turns out to be lung tumors. So it is not recommended to apply CT or PET scans in every such case. Biopsy of the nodules via transthoracic needle or surgical resection is the "gold standard" diagnostic test and can confirm if SPNs are cancerous or not, but this approach is invasive, potentially risky $(2,4)$. Specifically, surgery should be avoided in case of benign SPNs growths. Overall, it is clinically important to develop some new diagnostic strategies with high accuracy that are able to detect lung cancer in patients with SPNs in a minimally invasive manner.

Over years, a number of lung cancer-related markers have been identified, but few have turned out to be clinically relevant for the early diagnosis of lung cancer. It has been reported that sex hormones influence almost every aspect of human physiological functions, activities, and behaviors(12). Sex hormones are thought to have a role in the etiology of some cancers; disturbance in sex hormones is an important feature of cancer pathophysiology(13). However, most studies have been done on sex hormone-sensitive cancers in reproductive organs such as breast, prostate, cervix and ovary. It has been increasingly recognized that sex hormones also have a significant influence on cancers in non-reproductive organs or tissues such as colon, lung and esophagus $(13,14)$. The altered circulating sex hormones have been observed in human lung carcinoma, suggesting that sex hormones contribute to the development and progression of lung tumor(12). But the available reports were seemingly conflicting and complex. The role of sex hormones involving lung cancer is yet to be elucidated.

The objective of this study was to measure circulating sex hormones in male patients with SPNs, with exploration of whether circulating sex hormones have the potential to function as biomarkers in identification of lung cancer among patients with SPNs.

\section{Methods}

\section{Patients and clinical samples}

A total of 45 male patients with benign SPNs (age range between 38-72) and 47 male patients with malignant SPNs (age range between 40-79) as well as 32 male healthy controls (age range between 39-69) were included in this study.

All patients didn't receive radiation, chemotherapy, or other medical treatment and gave their blood samples preoperatively. Controls were healthy volunteers with no obvious illness who agreed to provide blood samples, health information and written informed consent. Serum was separated from the blood samples, aliquoted and stored at $-80^{\circ} \mathrm{C}$ for future analysis. For histological analysis, lung tissue specimens were resected surgically and fixed in 10\% neutral formalin, embedded in paraffin, cut into $5 \mu \mathrm{m}$ sections, and stained with hematoxylin \& eosin.

All subjects gave their informed consent for inclusion before they participated in the study. The study was approved by appropriate ethics committees and institutional review boards, which followed the recommendations of the Declaration of Helsinki for biomedical research involving human subjects.

\section{Measurement of circulating sex hormones and CEA}

Serum levels of testosterone, estradiol, and progesterone that represent both male and female sex hormones were measured by using commercially available enzyme-linked immunosorbent assay (ELISA) kits (Phoenix Pharmaceuticals, INC, USA), according to manufacturer's instructions. Serum levels of CEA were also measured by ELISA kit (Phoenix Pharmaceuticals, INC, USA). The detection limits for testosterone, estradiol, and progesterone as well as CEA are $0.05 \mathrm{ng} /$ $\mathrm{ml}, 10 \mathrm{pg} / \mathrm{ml}, 0.06 \mathrm{ng} / \mathrm{ml}$, and $1.0 \mathrm{ng} / \mathrm{ml}$, respectively.

\section{Determination of serum TNF- $\alpha$, IL- $\beta$ 1and IL-6 levels}

Serum samples were analyzed for TNF- $\alpha, \mathrm{IL}-1 \beta$ and IL-6 levels using ELISA kits (R\&D Systems Inc, USA), according to manufacturer's instructions. 


\section{Statistical analysis}

Data were expressed as mean \pm standard deviation (SD) and were analyzed by one-way analysis of variance (ANOVA). Results were considered statistically significant when $\mathrm{P}<0.05$. All statistical analyses were calculated using Graph Pad Prism.

\section{Results}

\section{Patient characteristics}

All patients had been diagnosed and staged either as malignant SPNs (lung squamous cell carcinoma or adenocarcinoma) or benign SPNs by a histological evaluation of surgical specimens (Supplementary Figure 1).

Supplementary Figure 1. Representative microscopic images of human patients with SPNs. (A) Lung paraneoplastic tissue; (B) Benign solitary pulmonary nodule; (C) Lung adenocarcinoma; (D) Lung squamous cell carcinoma. Lung tissues were resected by surgery from patients and fixed in 10\% formalin and embedded in paraffin wax. Then blocks were sectioned into $5 \mu \mathrm{m}$ thickness and stained with hematoxylin and eosin (H\&E) using a standard protocol. Scale bars: $100 \mu \mathrm{m}$.

\section{Supplementary Figure 1}

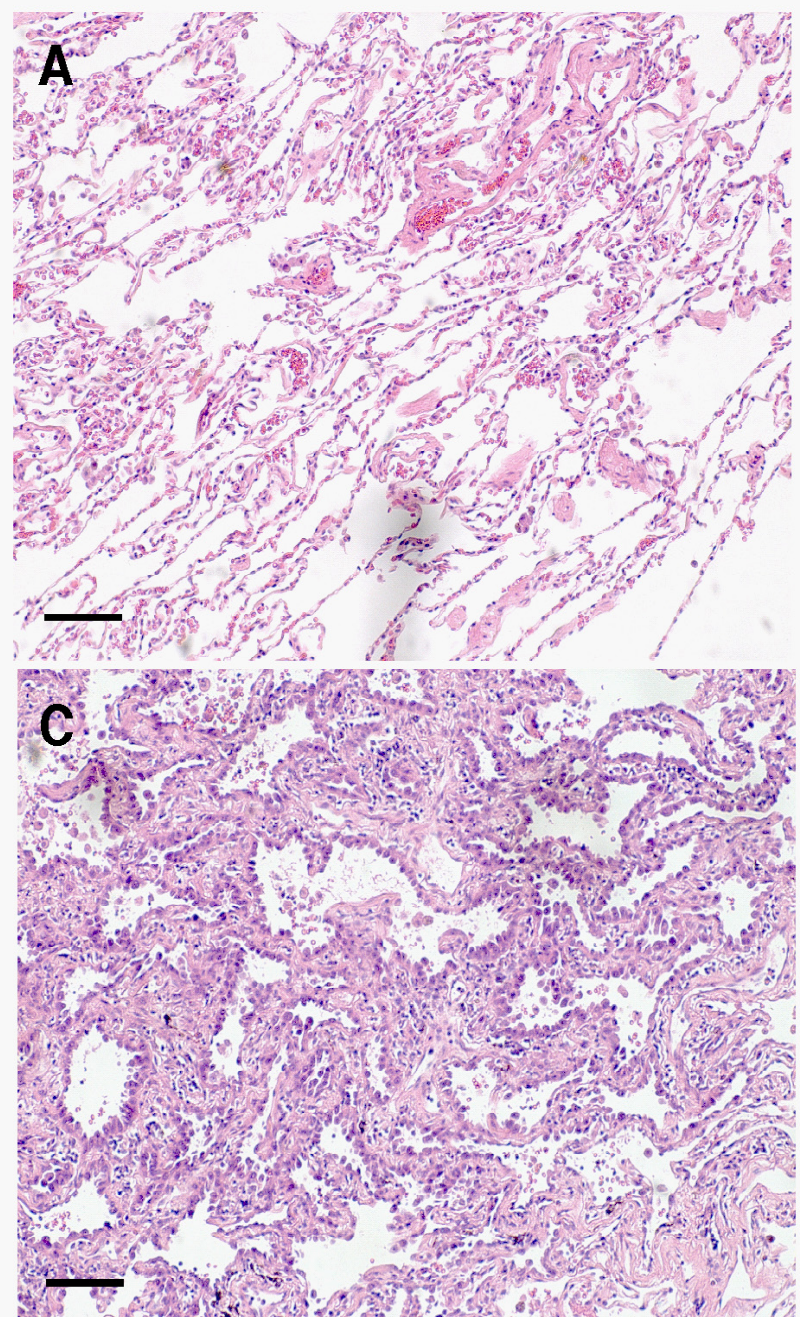

There were no statistical differences regarding the baseline demographic variables among the three groups, so all the subjects were comparable. For malignant SPNs, there were 29 cases of squamous carcinoma and 18 cases of adenocarcinoma, which were all at an early stage of
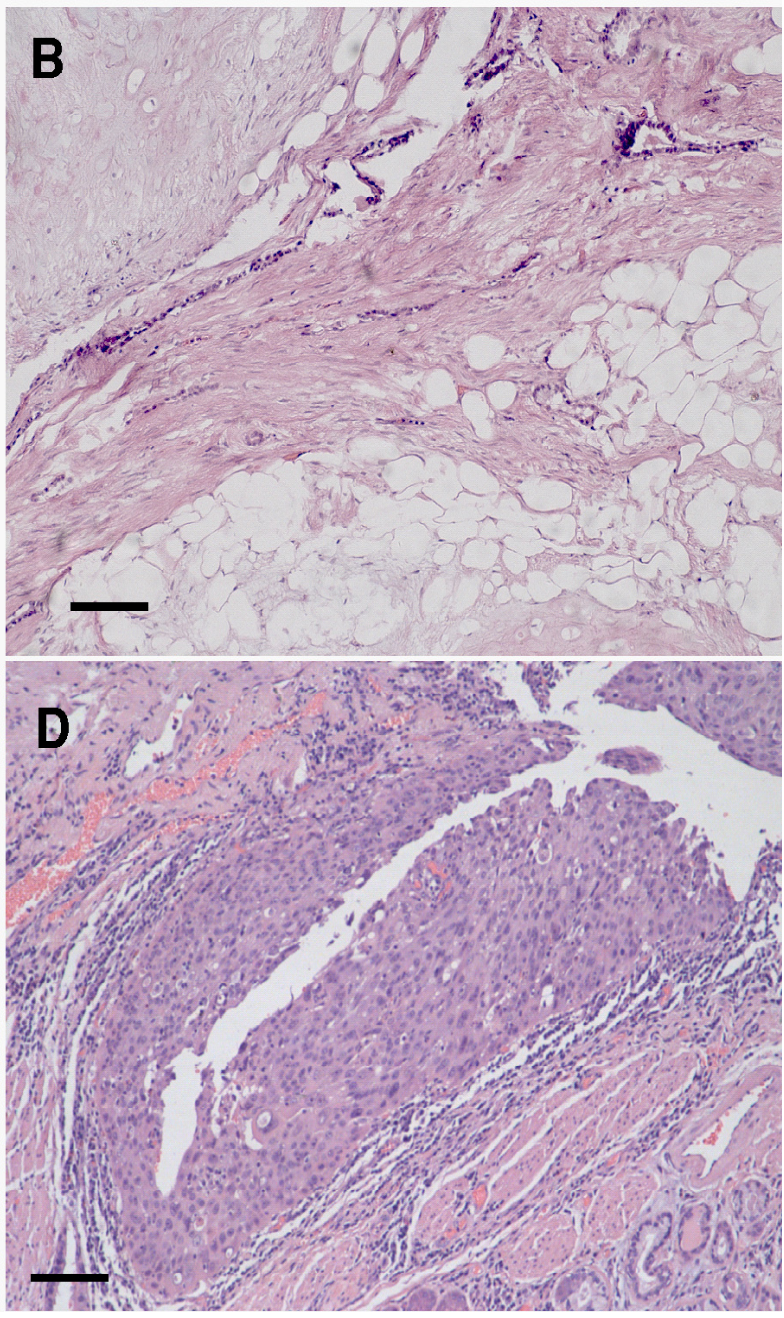

lung cancer (stage I). The benign SPNs were associated with various causes including infection, hamartoma, granuloma, and fibrosis. The detailed information of patients and healthy subjects was summarized in Table 1. 
Table 1. Characteristics of patients with benign solitary pulmonary nodules (SPNs), patients with malignant SPNs, and healthy controls

\begin{tabular}{llll}
\hline & Healthy controls & $\begin{array}{l}\text { Patients with benign } \\
\text { SPNs }\end{array}$ & $\begin{array}{l}\text { Patients } \\
\text { malignant SPNs }\end{array}$ \\
\hline No. of subjects & 32 & 45 & 47 \\
Gender & Male & Male & Male \\
Age range (median) & $39-69(57)$ & $38-72(58)$ & $40-79(62)$ \\
Active smokers (n) & 21 & 37 & 39 \\
Nodule size (average) & & $1.9 \mathrm{~cm}$ & $2.4 \mathrm{~cm}$ \\
Diagnosis of benign SPNs & & & \\
Inflammatory lesions & & 18 & \\
Hamatoma & 8 & \\
Granuloma & 9 & \\
Others & & \\
Histological types & & 29 \\
Adenocarcinoma & & \\
Squamous cell carcinoma & & \\
Cancer stage & & & \\
\hline
\end{tabular}

Circulating levels of sex hormones were decreased in patients with malignant SPNs

In this study, we measured both male and female sex hormones (testosterone, estradiol, and progesterone) in the serum of all patients and health subjects. Testosterone is the predominant form of androgen, whereas estradiol and progesterone are the principle forms of estrogen. We found that testosterone
(3.116 $\pm 0.48 \mathrm{ng} / \mathrm{ml})$, estradiol $(23.55 \pm 2.34 \mathrm{pg} / \mathrm{ml})$, and progesterone $(0.261 \pm 0.048 \mathrm{ng} / \mathrm{ml})$ in the serum of patients with malignant SPNs were all significantly lower than those in patients with benign SPNs $(4.701 \pm 0.49$ $\mathrm{ng} / \mathrm{ml}, 31.52 \pm 2.774 \mathrm{pg} / \mathrm{ml}, 0.451 \pm 0.052 \mathrm{ng} / \mathrm{ml})$ and healthy subjects $(5.581 \pm 0.372 \mathrm{ng} / \mathrm{ml}, 33.15 \pm 3.43 \mathrm{pg} /$ $\mathrm{ml}, 0.57 \pm 0.064 \mathrm{ng} / \mathrm{ml})($ all $\mathrm{p}<0.05$, Figure 1$)$.

The levels of three sex hormones tended to decline in

Figure 1. Circulating sex hormones were decreased in male patients with malignant SPNs. Serum levels of (A) testosterone (B) estradiol (C) progesterone were all significantly decreased in male patients with malignant SPNs, as compared with benign SPNs as well as normal controls. There was no significant difference in the levels of three sex hormones between patients with benign SPNs and controls $(\mathrm{P}>0.05)$.

\section{Figure 1}

\section{A}

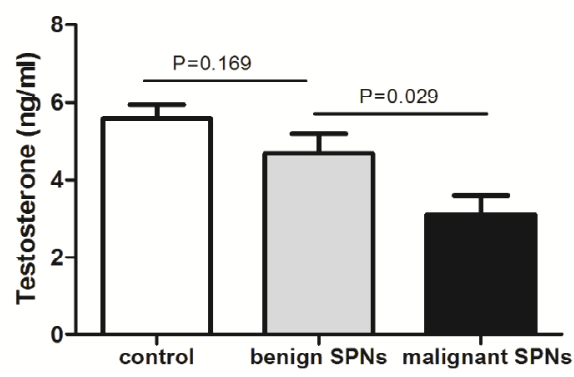

B

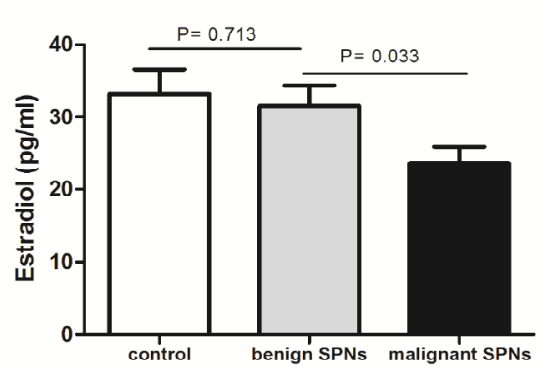

C

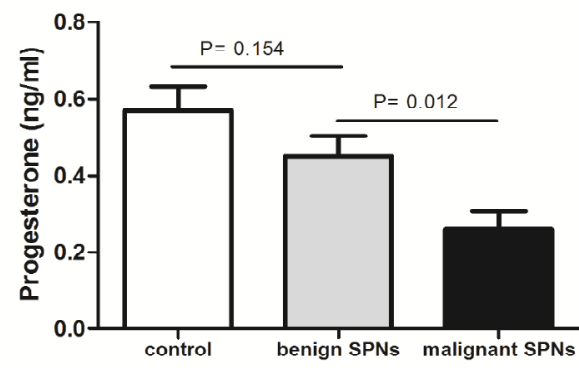


patients with benign SPNs in contrast to healthy subjects, but the difference was not statistically significant $(\mathrm{P}>0.05)$. In addition, there was no difference found in the levels of sex hormones between adenocarcinoma and squamous carcinoma of SPNs ( $P>0.05$, Supplementary Figure 2), indicating that sex hormones levels are not related to lung cancer histological types.

Supplementary Figure 2. Serum levels of sex hormones were similar in two types of lung cancer. There was no significant difference in the levels of (A) testosterone (B) estradiol (C) progesterone in patients with lung adenocarcinoma and patients with lung squamous carcinoma.

\section{Supplementary Figure 2}

A

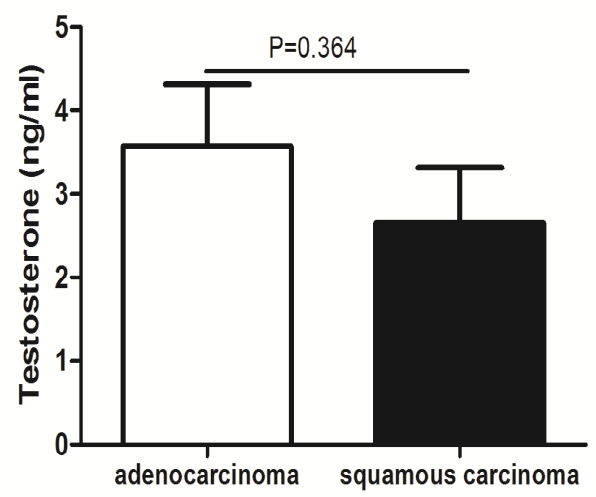

B

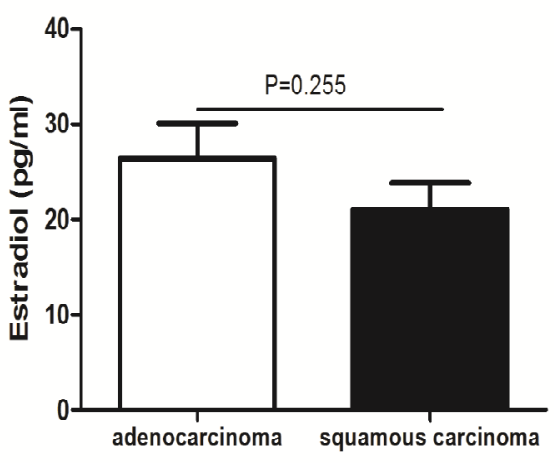

C

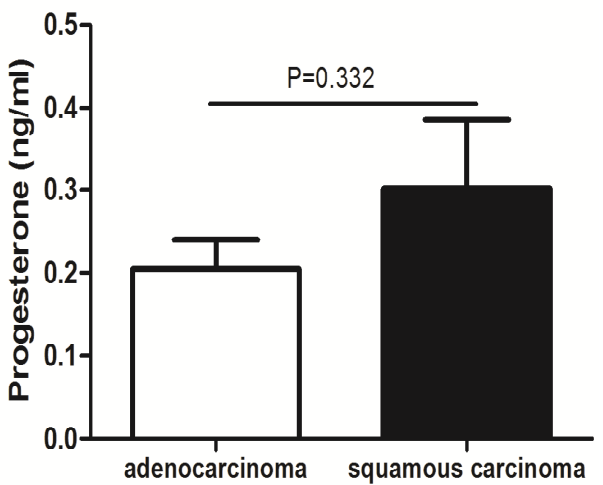

Serum levels of CEA were found to be abnormally elevated in only eight patients with lung adenocarcinoma, which were above the upper limit of normal value
(Supplementary Table 1). No abnormal elevation of CEA levels was observed in patients with lung squamous carcinoma and patients with benign SPNs.

Supplementary Table 1. Abnormal elevated serum levels of CEA in 8 male patients with lung adenocarcinoma

\begin{tabular}{llrccc}
\hline Case & Age (years) & Smoking status & Cancer stage & CEA $(\mathrm{ng} / \mathrm{ml})$ & Normal range $(<5.0 \mathrm{ng} / \mathrm{ml})$ \\
\hline Case1 & 54 & Current & I & 25.4 \\
Case1 & 48 & Current & I & 47.3 \\
Case3 & 71 & Former & I & 35.8 \\
Case4 & 67 & Current & I & 48.2 \\
Case5 & 44 & Current & I & 8.2 \\
Case6 & 49 & Current & I & 18.6 \\
Case7 & 58 & Current & I & 14.2 \\
Case8 & 65 & Current & I & 31.3 \\
\hline
\end{tabular}


Circulating levels of the inflammatory factors were increased in patients with SPNs

It showed that the levels of IL-1 $\beta(1.813 \pm 0.247 \mathrm{pg} /$ $\mathrm{ml}$ or $2.173 \pm 0.327 \mathrm{pg} / \mathrm{ml})$, IL-6 $(22.57 \pm 3.77 \mathrm{pg} / \mathrm{ml}$ or $27.37 \pm 3.686 \mathrm{pg} / \mathrm{ml})$, and TNF- $\alpha(11.73 \pm 2.349 \mathrm{pg} /$ $\mathrm{ml}$ or $10.96 \pm 2.257 \mathrm{pg} / \mathrm{ml}$ ) in the serum of patients with benign SPNs or malignant SPNs were remarkably higher than those in healthy subjects $(0.748 \pm 0.073$ $\mathrm{pg} / \mathrm{ml}, 2.615 \pm 0.243 \mathrm{pg} / \mathrm{ml}$, and $2.018 \pm 0.265 \mathrm{pg} / \mathrm{ml})$ (Figure 2).

Figure 2. Serum levels of the inflammatory factors were increased in patients with SPNs. (A) IL-1 $\beta$ (B) IL-6 (C) TNF- $\alpha$ levels were all increased in both patients with benign or malignant SPNs, as compared to controls. There was no significant difference in the levels of the inflammatory factors between patients with benign SPNs and patients with malignant SPNs $(\mathrm{P}>0.05)$.

\section{Figure 2}

A

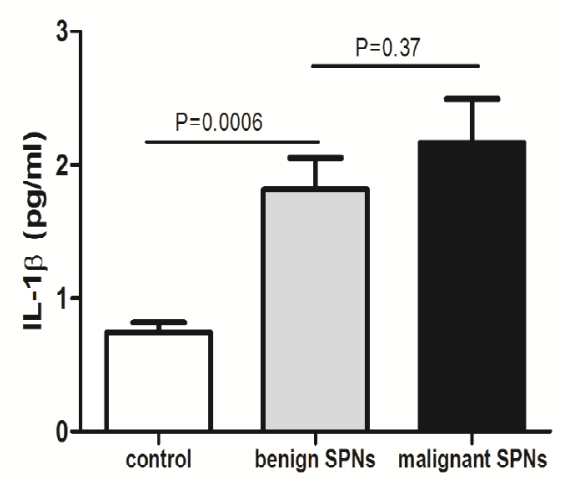

B

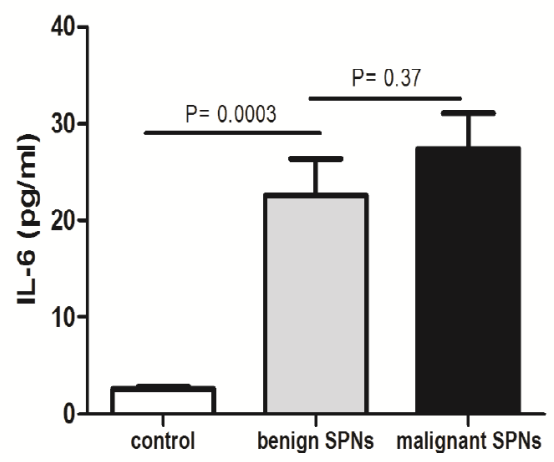

C

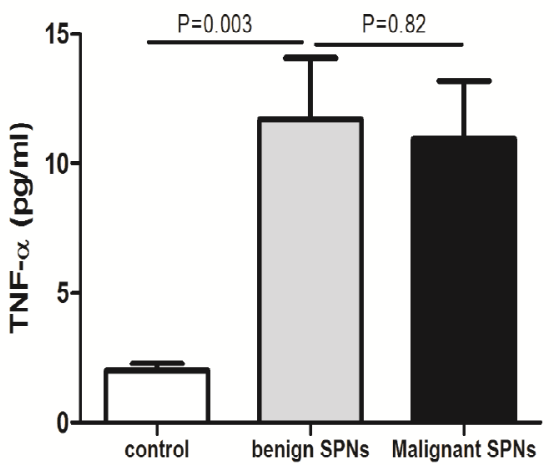

But there was no statistical difference in the levels of the inflammatory cytokine between patients with malignant or benign SPNs (P>0.05). It thus suggested that measurement of the inflammatory factors was unable to differentiate malignant from benign SPNs in patients.

\section{Discussion}

Serum measurement of sex hormones is a valuable index in evaluating a variety of organ dysfunctions, including reproductive and non-reproductive organ or tissues(13). Sex steroid hormones are primarily synthesized in the gonads, adrenal glands, and the feto-placental unit. Both male and female hormones are products of oxidative metabolism of cholesterol and their biosynthetic pathways are closely interrelated(15). Testosterone is the most important androgen and plays key roles in health and well-being in both males and females(16). Likewise, estradiol and progesterone are female hormones and essential for a variety of physiological processes in both males and females(17). In addition to their centrality in sex development, sex hormones have significant effects on various organs or tissues other than reproductive system including skin, blood vessels, bone, muscle, coagulation, gastrointestinal tract, brain, lung, liver, kidney, and pancreas. Alterations of circulating sex hormones have been implicated in a wide range of diseases(13). It is important to note that all sex steroid hormone receptors are found to be expressed in lung tissue(13), thus implying that sex hormones are closely associated with lung function and diseases. But the exact role of sex hormones in the pathogenesis and progression of lung cancer remains unclear, and there are actually some dramatic discrepancies in publishing reports. For example, certain studies suggested that androgens such as testosterone were decreased whereas estrogens such as estradiol were increased in patients with lung cancer $(12,18)$. But in other studies, there were no such associations observed. Even the levels of estradiol were reported to be decreased in patients with lung cancer(19). These confounding reports may be partly explained by gender differences in lung tumor patients caused by the variations in sex hormones levels in female patients. In addition, sex hormones levels may 
vary in different cancer stages.

It is a clinical challenge to distinguish malignant from benign SPNs using the current imaging technologies. Given that sex hormones play important but distinctive roles in the etiology of various lung diseases, we investigated the possibility of sex hormones as a diagnostic marker for the screening of lung cancer in patients with SPNs. Sex hormones levels differ in gender, especially in the case of women of childbearing age, pregnancy status and menopause. Furthermore, the levels of sex hormones decrease with age(13). To rule out these potential confounders, we only collected male, age-matched patients with benign or malignant SPNs in this study. Moreover, malignant SPNs were confirmed to be an early stage of lung cancer (stage I), thus excluding the possible interfering factor involving cancer stage.

Our study showed that circulating sex hormones (testosterone, estradiol, and progesterone) were found to be decreased remarkably in patients with malignant SPNs. This seemed to be very unique and solely associated with lung cancer because the trend was not observed in patients with benign SPNs as compared to controls. To further explore whether the changes of sex hormones related to lung cancer are distinctive, we also measured the levels of CEA and the inflammatory cytokines in the serum of all patients. It is aware that the increased levels of CEA are related to caner stage and extent of the lung, liver, breast, colon, and prostate(20). But it showed that only eight cases of lung adenocarcinoma patients had abnormally elevated levels of CEA, suggesting that this marker is not ideal for differentiation between malignant and benign SPNs. Similarly, the inflammatory cytokines were found to be elevated significantly in both patients compared to normal controls, but there was no statistical difference between patients with benign or malignant SPNs. Major causes of benign SPNs include chronic smoking, granuloma, and infections, which could trigger inflammatory responses(4). So it is not a surprise that we observed high levels of inflammatory cytokines in patients with benign SPNs. Likewise, patients with malignant SPNs had higher levels of inflammatory cytokines, suggesting that inflammation also occurred during the course of lung tumorigenesis. It suggests that the inflammatory biomarkers are unable to distinguish lung cancer patients from benign SPNs patients.

The mechanism that circulating sex hormones were reduced significantly in male patients with malignant SPNs is not clear. Indeed, sex hormones are involved in a wide-ranging and complex modulation of cellular function in the lung. Under normal conditions, both androgen (testosterone) and estrogens (estradiol, progesterone) are proposed to have beneficial effects to modulate lung structure and function(13). For example, androgen enhances the inhibition of immune response and stimulate the growth of lung tissue(21), whereas estrogens participatein the regulation of manypulmonary functions such as pulmonary vasodilation $(17,19)$. We speculate that disorders of lung function as a result of lung tumorigenesis may be the reason for the reduced sex hormones in patients. For benign SPNs, they may exert weak or little influence on sex hormones levels in the circulation. More rigorous studies are needed to clarify the underlying mechanisms.

\section{Conclusions}

Based on our study, it showed that measurement of circulating sex hormones in the serum of male patients with SPNs may have clinical potential to help distinguish malignant from benign SPNs. Considering that these patients with malignant SPNs were all at an early stage of lung cancer, the changes of sex hormones levels could be used as biomarkers for an early differentiation. In all, our finding is intriguing and worth further exploration. But it is a preliminary study and our speculative hypotheses require further investigation in larger population studies.

\section{Conflict of interest}

The authors have declared that no competing interests exist.

\section{Acknowledgements}

This work was supported by National Natural Science Foundation of China (81272142) to Dr Xin Lv and in part by National Natural Science Foundation of China (81372094) to Dr Tao Wen.

\section{References}

1. Brandman S, Ko JP. Pulmonary nodule detection, characterization, and management with multidetector computed tomography. Journal of thoracic imaging. 2011;26(2):90-105.

2. Ost D, Fein A, Feinsilver S. The solitary pulmonary nodule. New England Journal of Medicine.2003;253542.

3. Gould MK, Fletcher J, Iannettoni MD, Lynch WR, Midthun DE, Naidich DP, et al. Evaluation ofpatients with pulmonary nodules: when is it lung cancer?: ACCP 
evidence-based clinical practice guidelines (2nd edition). Chest. 2007;132(3 Suppl):108S-130S.

4. MacMahon H, Austin JHM, Gamsu G, Herold CJ, Jett JR, Naidich DP, et al. Guidelines for managementof small pulmonary nodules detected on CT scans: a statement from the Fleischner Society. Radiology. 2005;237(2):395-400.

5. Libby DM. Managing the Small Pulmonary Nodule Discovered by CT. Chest. 2004;125(4):1522-9.

6. McCarville MB, Lederman HM, Santana VM, Daw NC, Shochat SJ, Li C-S, et al. Distinguishing benignfrom malignant pulmonary nodules with helical chest CT in children with malignant solid tumors. Radiology. 2006;239(2):514-20.

7. Jemal A, Bray F, Center MM, Ferlay J, Ward E, Forman D. Global cancer statistics. CA: a cancer journalfor clinicians. 2011;61(2):69-90.

8. Shen J, Liu Z, Todd NW, Zhang H, Liao J, Yu L, et al. Diagnosis of lung cancer in individuals withsolitary pulmonary nodules by plasma microRNA biomarkers. BMC cancer. 2011;11(1):374.

9. Ohno Y, Koyama H, Matsumoto K, Onishi Y, Takenaka D, Fujisawa Y, et al. Differentiation ofmalignant and benign pulmonary nodules with quantitative firstpass 320-detector row perfusion CTversus FDG PET/ CT. Radiology. 2011;258(2):599-609.

10. Gao L, Wen Z, Wu C, Wen T, Ong C. Metabolic Profiling of Human Benign and Malignant PulmonaryNodules Using Mass Spectrometry-Based Metabolomics. Metabolites. 2013;3(3):539-51.

11. Bunyaviroch T, Coleman RE. PET evaluation of lung cancer. Journal of nuclear medicine.2006;47(3):451-69.

12. Folkerd EJ, Dowsett M. Influence of sex hormones on cancer progression. Journal of clinical oncology :official journal of the American Society of Clinical On- cology. 2010;28(26):4038-44.

13. Townsend E a., Miller VM, Prakash YS. Sex Dif-

ferences and Sex Steroids in Lung Health and Disease. Endocrine Reviews. 2012;33(1):1-47.

14. Tam A, Morrish D, Wadsworth S, Dorscheid D, Man SFP, Sin DD. The role of female hormones onlung function in chronic lung diseases. BMC women's health; 2011;11(1):24.

15. Veldhuijzen DS, Keaser ML, Traub DS, Zhuo J, Gullapalli RP, Greenspan JD. The role of circulatingsex hormones in menstrual cycle-dependent modulation of pain-related brain activation. Pain. 2013;154(4):548-59. 16. Hyde Z, Flicker L, McCaul Ka, Almeida OP, Hankey GJ, Chubb S a P, et al. Associations betweentestosterone levels and incident prostate, lung, and colorectal cancer. A population-based study.Cancer epidemiology, biomarkers \& prevention. 2012;21(8):1319-29.

17. Tam A, Morrish D, Wadsworth S, Dorscheid D, Man SFP, Sin DD. The role of female hormones onlung function in chronic lung diseases. BMC women's health. 2011;11(1):24.

18. Pikwer M, Giwercman A, Bergström U, Nilsson J-A,, Jacobsson LTH, Turesson C. Association betweentestosterone levels and risk of future rheumatoid arthritis in men: a population-based case-controlstudy. Annals of the rheumatic diseases. 2013;1-7.

19. Thomas L, Doyle LA, Edelman MJ. Lung cancer in women: emerging differences in epidemiology,biology, and therapy. Chest. 2005;128(1):370-81.

20. Grunnet M, Sorensen JB. Carcinoembryonic antigen (CEA) as tumor marker in lung cancer. Lungcancer. 2012;76(2):138-43.

21. Nishio M, Ohyanagi F, Horiike a, Ishikawa Y, Satoh Y, Okumura S, et al. Gefitinib treatment affectsandrogen levels in non-small-cell lung cancer patients. British journal of cancer.2005;92(10):1877-80. 